\title{
Aproximación a un estudio sobre las lesiones del bahareque en el estado Zulia, Venezuela. Algunas recomendaciones para su intervención.
}

\author{
Approach to a study of bahareque damages in Zulia state, \\ Venezuela. Some recommendations for intervention actions.
}

\author{
A. Henneberg de León ${ }^{(*)}$
}

RESUMEN

El bahareque es una técnica constructiva formada por cuatro componentes hechos de materiales diversos: horconadura, enlatado, relleno y empañetado. Estos componentes sufren diferentes lesiones, por lo que para su intervención son requeridas distintas soluciones para cada uno. Este artículo presenta los primeros resultados de una investigación de campo que estudió las lesiones más frecuentes que sufren dichos componentes, al igual que propone recomendaciones para su intervención, basadas en rehabilitaciones hechas en el Estado Zulia y en la construcción de una pared de bahareque. Se pudo determinar que la mayor cantidad de lesiones son físicas y mecánicas y se producen en el empañetado, componente expuesto al ambiente. Los otros componentes, se lesionan solo cuando quedan al descubierto. Este estudio intenta servir de base para futuros estudios sobre la patología del bahareque; además, de contribuir a la revalorización de esta técnica, pudiendo ser considerada como opción técnica en la construcción de viviendas.

$650-8$

Palabras clave: Bahareque, Acciones de intervención, Lesiones del Bahareque, Construcción con tierra, Patología de la construcción.

\section{SUMMARY}

The bahareque is a constructive technique made by four components of different materials: wood colums, canning, filling and mud-cover. These components suffer different damages, therefore when repairing these walls different solutions are required for each component. This article presents the first results of a field research, that studied the more frequent damages that undergo these components, and it proposes some recommendations for its repair, based on rehabilitations done in the Zulia State and in the construction of a baharequewall. It was possible to establish that the greater amount of damages was physical and mechanical and they occurred in the mud-cover, component exposed to the environment. The other components only deteriorate when they are uncovered. This study try to offer a source for future researches about the pathology of the bahareque; besides, it can contribute to revalue this technique, being even considered as a technical option for the construction of houses.

Keywords: Bahareque, Intervention actions, Bahareque damages, Earth construction, Construction pathology. 
1. Componentes del bahareque. Elaboración de la autora (2010).

\section{INTRODUCCIÓN}

El bahareque, aunque es una técnica constructiva precolombina, fue empleado en el Estado Zulia a partir del siglo XVII (1), para la construcción de viviendas y de algunas iglesias. Su uso se extendió hasta la segunda mitad del siglo XX, en donde dejó de utilizarse en las ciudades y su empleo quedó relegado para las viviendas de las zonas rurales. Debido al uso de de esta técnica durante casi cuatro siglos, todavía perduran en las ciudades edificaciones construidas con esta técnica, siendo algunas de ellas declaradas patrimonio regional o nacional.

El bahareque puede circunscribirse dentro de las construcciones de tipo entramado hechas con madera y barro. En el estado Zulia, el bahareque está compuesto por un conjunto de columnas de madera a las cuales son amarradas varas horizontales a ambos lados, de tal manera que queda conformada una cámara la cual se rellena con barro y otros materiales como piedras, conchas de coco, pedazos de ladrillo y/o paja. Luego es aplicado por ambas caras un acabado hecho de una mezcla con barro y agua con o sin cal, la cual al secarse se pinta con cal o con pintura látex de color. Se puede decir que el bahareque presenta cuatro partes o componentes: horconadura, enlatado, embutido o relleno y empañetado (Figura 1). Enlatar es "Formar una cerca con latas de madera" (2). A veces se dice en vez de enlatado, también encañado, cuando las latas son de caña. Para el término empañetado, a veces son usados, en otros países, términos como revoco, revoque, friso, pañete o repello. Estos términos implican mezclas que no siempre tienen barro como componente, mientras que el empañetado está siempre hecho a base de barro.

En esta técnica el entramado de madera tiene la función de soporte y el relleno de barro y piedras solo es usado para el cerramiento.

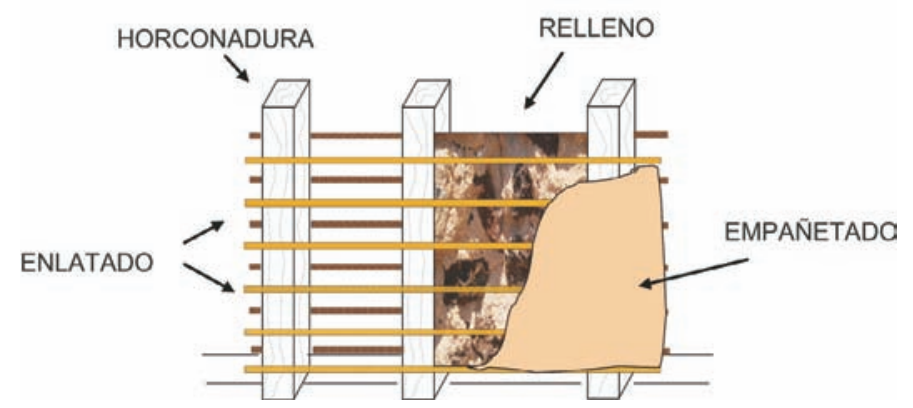

Debido a los diversos materiales que conforman esta pared como: madera (horcones), caña y bejucos (varas horizontales), piedra mezclada con barro y cal (relleno) y mezcla de barro (empañetado), se manifies- tan diversas lesiones a lo largo del tiempo. Por consiguiente, algunas de las edificaciones de bahareque en el Estado Zulia que subsisten, se encuentran en un estado de conservación regular, y otras en mal estado. Los estudios sobre la patología de esta técnica constructiva en Venezuela son escasos. La mayoría de las investigaciones o publicaciones sobre el tema, son estudios sobre las características del bahareque (3), propuestas para su mejoramiento técnico (4), (5) o propuestas de intervenciones de edificaciones específicas (6), (7). El vacío en esta temática, ha sido una de las razones por la cual se acometió una investigación, como parte de la tesis doctoral de la autora (1), en la cual se deseaba determinar todas las posibles lesiones que aparecen en el bahareque, con la finalidad de aportar conocimiento al tema, y a la vez ofrecer una base para futuras investigaciones y para las intervenciones de estas edificaciones. El ámbito de estudio escogido fue el estado Zulia, con un alcance muy general sobre las lesiones y sus posibles causas, como una primera aproximación al estudio de la patología constructiva del bahareque. Además, se esperaba proponer algunas recomendaciones técnicas y constructivas para su reparación, rehabilitación u otro tipo de intervención.

\section{OBJETIVOS}

Este artículo plantea los primeros resultados de esta investigación sobre las lesiones más frecuentes que sufren los diferentes componentes que conforman la pared de bahareque, al igual que presenta recomendaciones para la intervención de las edificaciones de bahareque del Estado Zulia. EI estudio sobre las lesiones es producto de un trabajo de campo, mientras que las recomendaciones de intervención fueron obtenidas de dos rehabilitaciones de edificaciones de bahareque realizadas en el Estado Zulia en los últimos años y de la construcción de un prototipo de bahareque que realizó la autora durante la investigación.

Por lo que este artículo tiene un doble propósito:

-Exponer de forma aproximada las lesiones más frecuentes que presentan los componentes del bahareque y sus posibles causas.

- Plantear algunas recomendaciones para la intervención de los componentes constructivos del bahareque.

\section{METODOlOGíA}

Con la finalidad de establecer cuáles son las lesiones más frecuentes y las causas que 
las producen, se emprendió un trabajo de campo, en el cual fueron visitados 20 poblados del Estado Zulia y recorridos una vía interestatal (Vía Lara-Zulia) y una vía extraurbana (Vía Intercomunal) (Figura 2).

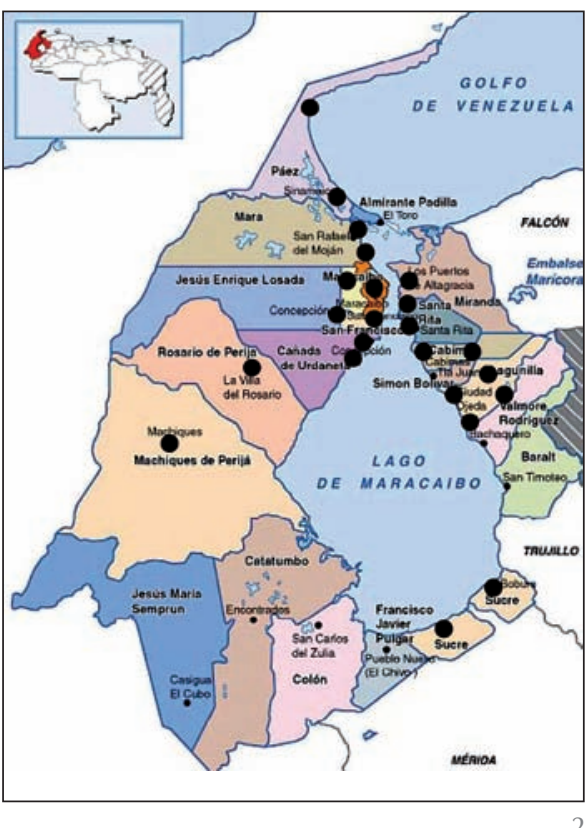

De estas visitas fue posible recoger datos de 26 viviendas en 11 ciudades o poblados del estado Zulia y en la Vía Lara-Zulia. Los poblados fueron: Maracaibo (9 viviendas), La Guajira (1 vivienda), Cabeza de Toro (1 vivienda), La Cañada (1 vivienda), El Carmelo (1 vivienda), El Bajo (1 vivienda), San Francisco (1 vivienda), Puerto Escondido (1 vivienda), Puertos de Altagracia (3 viviendas), Bobures (1 vivienda), San Antonio ( 1 vivienda y 1 capilla) y Vía LaraZulia (3 viviendas). En los poblados visitados de San Isidro, Machiques, Santa Cruz de Mara, La Villa de Rosario, Cabimas, San Rafael del Moján y a lo largo de la vía Intercomunal, las edificaciones de bahareque estaban cerradas o eran inaccesibles, y en Sinamaica, La Concepción y Ciudad Ojeda, no habían edificaciones de bahareque.

Debido al bajo número de viviendas inspeccionadas, los resultados solo pueden considerarse de forma aproximada. Se recomienda continuar en el futuro con la toma de datos de mayor cantidad de viviendas.

Para la toma de datos fue diseñada una ficha (Tabla 1) la cual fue aplicada a cada edificación, al igual que se realizó un registro fotográfico de cada lesión. Para el Ilenado de las fichas se utilizó la inspección visual. En cada ficha, se recogió información sobre todas las lesiones que aparecen en la edificación, el componente constructivo en el cual aparece y la posible causa. Esta ficha contiene los siguientes descriptores:
Datos de identificación: Código de identificación de la ficha, localización (poblado) de la edificación, dirección de la edificación, ubicación en área urbana, semi-urbana o rural, orientación de la edificación y estado de conservación (bueno, regular, malo, ruina).

Tipos de lesiones: Las lesiones fueron agrupadas de acuerdo a su naturaleza, en físicas, mecánicas y químicas, tal como las clasifica Monjo Carrió (8), escogiendo para esta ficha las siguientes lesiones:

- Lesiones físicas: Las humedades (de origen freático, capilar, por filtración, por condensación y accidentales), la suciedad (por depósito y por lavado diferencial) el salpiqueo, la erosión atmosférica, la resequedad de la madera y el envejecimiento de madera (decoloración y fendas). Estas dos últimas son lesiones que se presentan en la madera producidas por los agentes atmosféricos (9).

-Lesiones mecánicas: Las grietas, las fisuras, los desprendimientos (acabado continuo y acabado por elementos), los descascaramientos, la erosión mecánica, las deformaciones y las roturas.

-Lesiones químicas: Las eflorescencias, los organismos (vegetales y animales), la oxidación, la erosión química (ampollas y farinage) y la desintegración del material.

Datos de los componentes del bahareque: Fueron desglosados los componentes del bahareque en: fundación (horcón enterrado), horconadura, enlatado, relleno y empañetado, agregando las uniones entre las varas y las varas con el horcón.

Causas de la lesión: Las posibles causas fueron agrupadas en intrínsecas y extrínsecas (10).

-Causas intrínsecas: Son aquellas producidas por el material utilizado más que por las características de la técnica en sí, como:

- Propias del material: El barro, las cañas y la madera son materiales susceptibles a dañarse por efecto los agentes ambientales. También hay que considerar que cualquier defecto que tenga el material (madera, caña) puede a la larga producir lesiones a toda la pared de bahareque.

- Fallos en los procesos constructivos: Si ha habido alguna negligencia o alguna falla durante el proceso constructivo, lo más probable es que empiece a deteriorarse por efecto de la propia falla.

- Disposición del elemento en el conjunto: Un elemento mal ubicado. Por ejemplo, una columna no alineada o una vara mal amarrada.
2. Mapa del estado Zulia con los poblados visitados.

Elaboración de la autora (2011). 
-Causas extrínsecas: Son aquellas provenientes del contexto en el cual se ubica la pared y del uso que le es asignado a la edificación. Pueden ser:

- Humedades en general: Las humedades en general pueden originar otras lesiones como la presencia de organismos, desprendimientos de los acabados y la eflorescencia.

- Agresiones ambientales: El viento, el sol y las lluvias pueden ser muy nocivos para estas paredes por su acción erosiva. La existencia de aleros menores de $30 \mathrm{cms}$ o su total ausencia en estas edificaciones contribuye a que las paredes se encuentren completamente expuestas a las lluvias incrementándose de esta forma el tenor de humedad en ellos. También el salpiqueo que se genera cuando la lluvia golpea la acera o el piso, daña a la larga la parte inferior de las paredes. En la ficha se colocó por separado las Iluvias como causantes del salpiqueo y del lavado diferencial.

- Agresiones humanas: Las personas pueden causar lesiones de forma intencional o accidental.

- Agentes bióticos: La presencia de animales (ratas, palomas, etc) y de insectos xilófagos pueden deteriorar la madera, las varas y los empañetados.

- Falta de mantenimiento: Cualquier pared hecha de tierra debe ser mantenida apropiadamente.
- Reparaciones inadecuadas: El uso indiscriminado del cemento para reparar grietas y desprendimientos de los empañetados, aumenta las lesiones debido a que las proporciones de cemento utilizadas son incompatibles con las mezclas originales de las paredes.

- Problemas estructurales: El uso de maderas o materiales con resistencias insuficientes a los esfuerzos a los cuales estarán sometidos, puede causar pandeos y/o roturas en los elementos estructurales lo que a su vez conlleva a lesiones o desplomes de las paredes.

Fotografías: Como apoyo a estos datos se incluyeron fotografías de algunas lesiones.

Observaciones: Cualquier otra observación importante que se desee colocar.

\section{ANÁLISIS DE LOS RESULTADOS DEL TRABAJO DE CAMPO}

Después de haber aplicado la ficha y recogido la información de las 26 edificaciones inspeccionadas, se procedió a organizar y analizar los datos haciendo para ello un cuadro general con toda la información obtenida. En dicho cuadro aparece la cantidad de edificaciones que padecían cada una de las lesiones de acuerdo a cada componente

Tabla 1

Ficha sobre la patología de viviendas de bahareque

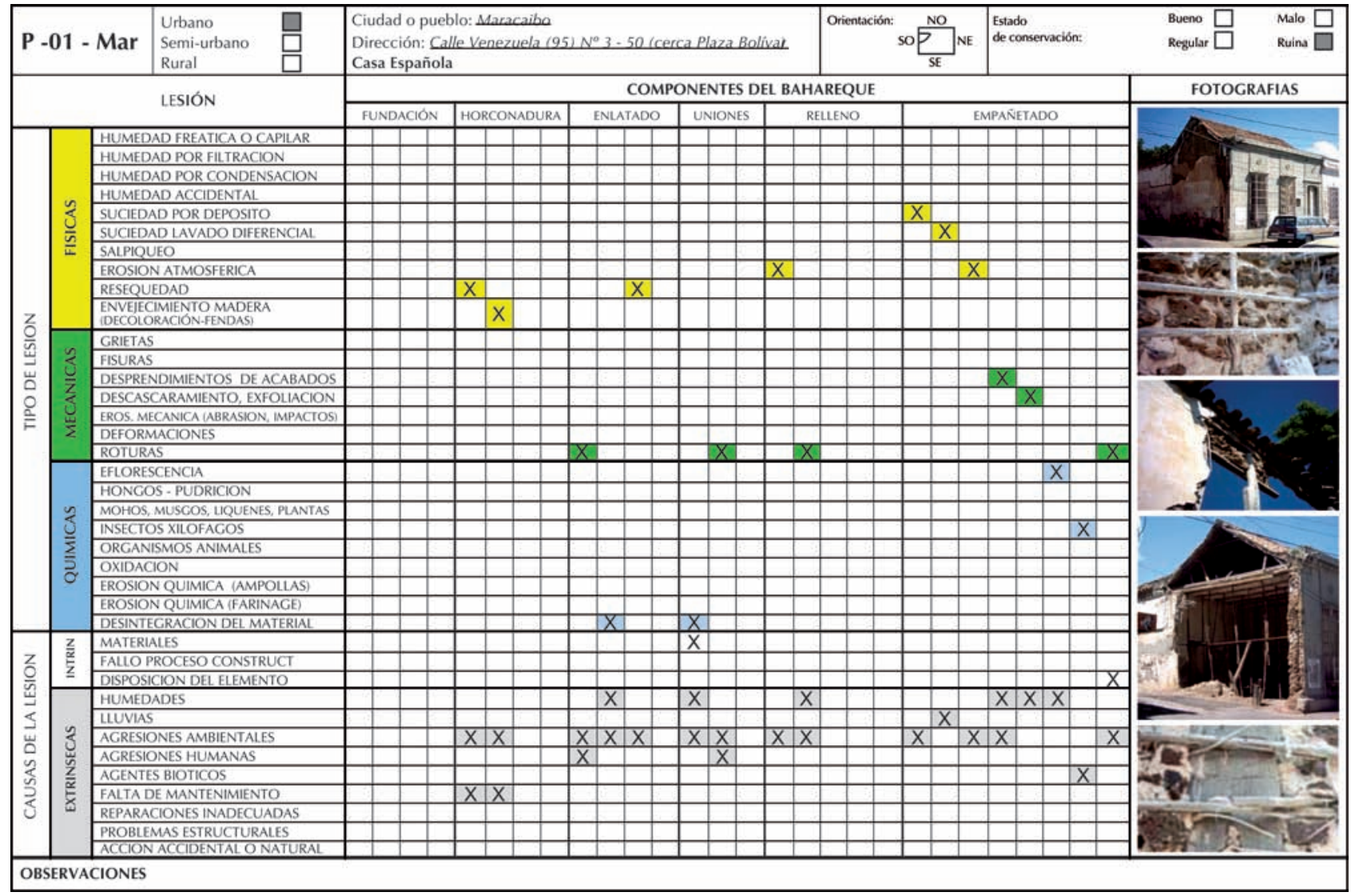


del bahareque, y la cantidad de edificaciones que coinciden con la causa probable de cada lesión. A cada lesión aparecida en cada componente constructivo del bahareque, fue aplicado un cálculo porcentual expresándolo en gráficos de barra, para así obtener las lesiones más frecuentes y menos frecuentes.

También se pudo determinar que de las edificaciones inspeccionadas: nueve estaban en buen estado (Figura 3), cinco en regular estado (Figura 4), dos en mal estado (Figura 5 ) y diez en estado de ruina (Figuras 6,7 y 8 ).
- Envejecimiento de la madera (69\%). Se observaron fendas y una coloración gris de madera envejecida producida por las agresiones ambientales.

- Grietas (19\%). Se encontraron en los horcones que tuvieron problemas estructurales debido a esfuerzos excesivos.

-Hongos de pudrición (19\%). Fueron observados en horcones con humedad o que estaban en ambientes húmedos. En estos casos los hongos se alimentaron del interior de la madera disminuyendo así la capacidad portante del horcón.
3. Vivienda usada como intendencia en zona de Santa Lucía en Maracaibo, Estado Zulia. Archivo fotográfico de la autora (2009).

4. Vivienda en San Francisco, Estado Zulia.

Archivo fotográfico de la autora (2009).

5. Vivienda en Los Puertos de Altagracia, Estado Zulia.

Archivo fotográfico de la autora (2005).

6. Vivienda en la zona de La Plata-Vía Lara-Zulia.

Archivo fotográfico de la autora (2009).
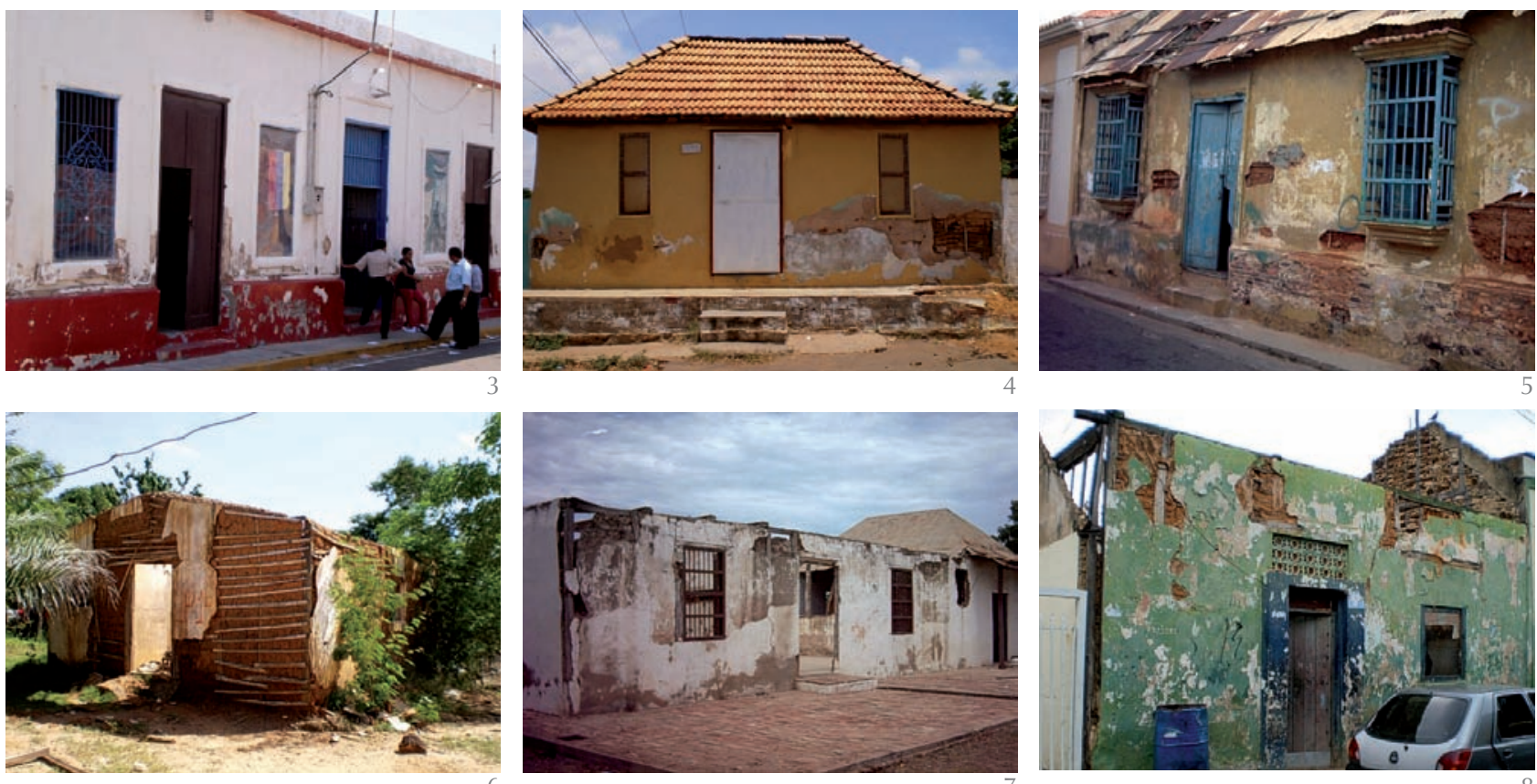

Las lesiones más frecuentes que padecen las edificaciones hechas de bahareque según el componente constructivo y su porcentaje de aparición, fueron las siguientes:

\subsection{Fundaciones (horcón enterrado)}

Solo se pudieron examinar dos edificaciones con fundaciones. La lesión aparecida en estas fundaciones fue:

- Desintegración del material por efecto de la humedad, proveniente de la humedad capilar o de filtración por falta de protección impermeabilizante de la madera, y posiblemente por agentes bióticos.

\subsection{Horconadura}

Los horcones que estaban al descubierto, debido al desprendimiento del empañetado, mostraron las siguientes lesiones.

- Resequedad (81\%). La madera estaba deshidratada (Figura 9). Es la lesión que más sufre la horconadura debido a las agresiones ambientales.

-Desintegración de la madera (19\%). En algunos casos se había desintegrado la parte exterior de la madera (Figura 9) y en otros había desaparecido completamente parte del horcón. Es una lesión producto de humedades y de los efectos del sol y el viento.

-Deformaciones (12\%). El horcón sufrió deformaciones porque los esfuerzos sobrepasaron su capacidad resistente o porque fue colocado ya deformado. No es una lesión muy usual, ya que los horcones generalmente son de madera muy resistente.

- Humedad capilar (4\%). Fue encontrada cierta humedad en la parte inferior del horcón, producto de un nivel freático muy alto o por humedad en el suelo. Esta lesión puede dar origen a otras lesiones.

- Insectos xilófagos (4\%). Se observaron rastros de insectos xilófagos (no se pudo determinar el tipo) que se alimentaron del horcón debilitándolo. No es una lesión muy frecuente por el tipo de madera usada que generalmente es dura.
7. Vivienda Natal de Rafael Urdaneta en El Carmelo, Estado Zulia, antes de su rehabilitación.

Archivo fotográfico de la autora (2007)

8. Vivienda en la zona de Veritas en Maracaibo, Estado Zulia. Archivo fotográfico de la autora (2009).

9. Horcón reseco y desintegrado en su parte inferior - Vivienda en Veritas, Maracaibo.

Archivo fotográfico de la autora (2009)

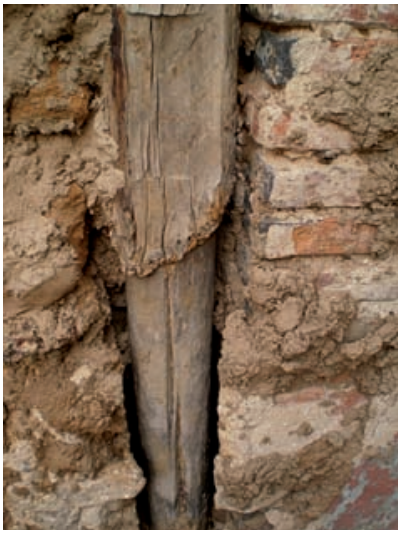


Las lesiones predominantes son de tipo físico (Figura 10).

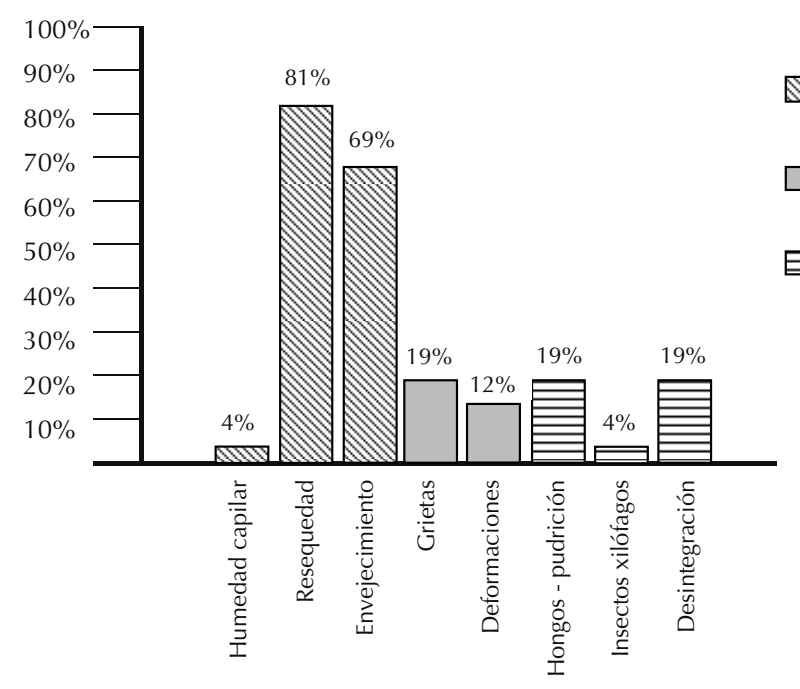

10

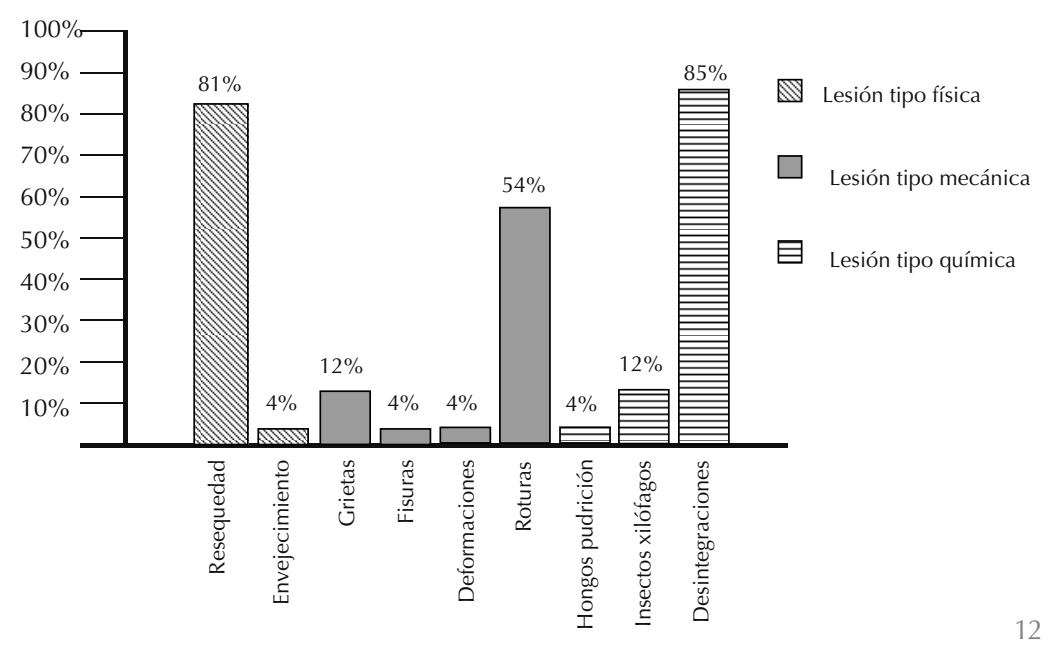

10. Lesiones aparecidas en la horcadura.

Elaboración de la autora (2010).

11. Desintegración del enlatado -Vivienda en La Plata, Vía Lara -Zulia.

Archivo fotográfico de la autora (2009).

12. Lesiones aparecidas en el enlatado.

Elaboración de la autora. nimiento. Los horcones no sufrieron por efectos de problemas propios del material, por el proceso constructivo o por la disposición del elemento. Tampoco se daña-
Las lesiones más frecuentes fueron:

-Desintegración del material (85\%). Es una lesión observada cuando el enlatado queda expuesto completamente. En este caso el enlatado no existe debido a las agresiones ambientales (Figura 11).

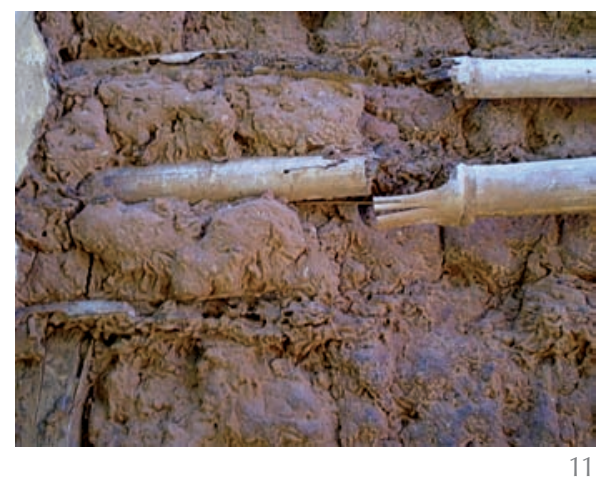

- Resequedad (81\%). Esta lesión apareció en aquellas varas expuestas a la intemperie.

- Roturas (54\%). Las varas se encontraron rotas debido a las acciones de la intemperie o porque los usuarios las rompieron.

- Grietas (12\%). Lesión producida debido a esfuerzos excesivos.

- Insectos xilófagos (12\%). Debido a un estado de humedad constante los insectos xilófagos atacaron el enlatado y lo degradaron.

-Envejecimiento (4\%). Al quedar el enlatado expuesto a la intemperie, este se fue envejeciendo por las acciones ambientales.

-Fisuras (4\%). Aparecieron producto de la acción de esfuerzos o por defecto del material.

-Deformaciones (4\%). Fue observado en algunos casos debido a que el enlatado estaba sometido a esfuerzos que superaban su capacidad resistente.

-Pudrición por hongos (4\%). Las varas del enlatado se pudrieron por efecto de hongos debido a un ambiente con mucha humedad.

Las lesiones predominantes son de tipo químico, como puede observarse en el siguiente gráfico de barras (Figura 12). ron por agresiones humanas, reparaciones inadecuadas o acciones accidentales o naturales.

\subsection{Enlatado}

Las varas que están protegidas por el empañetado se conservan en buen estado, pero cuando quedan expuestas al aire por el desprendimiento del empañetado se degradan casi completamente y pierden toda su capacidad resistente.
La causa que afectó mayormente el enlatado fueron las agresiones ambientales cuando el enlatado quedaba descubierto por el desprendimiento del empañetado. Luego siguieron las humedades y los problemas por el comportamiento estructural.

También presentó lesiones, aunque pocas, causadas por el material mismo, por el proceso constructivo, los agentes bióticos y la falta de mantenimiento. No presentó ningún deterioro por su disposición en la pared, por reparaciones inadecuadas o por acciones accidentales o naturales. 


\subsection{Uniones}

Aunque las uniones son parte del enlatado, estas pueden ser de diferentes materiales como: bejucos, fibras naturales, alambre de cobre, alambre dulce o clavos. Por la variedad de materiales usados, las uniones presentan lesiones diferentes al enlatado.

Las lesiones más comunes fueron las siguientes:

-Desintegración del material (54\%). En varios casos la unión hecha con bejuco era inexistente, es decir, había desaparecido, producto de las humedades y de las agresiones ambientales.

-Resequedad (27\%). Cuando el enlatado quedaba al descubierto, la acción del viento y del sol deterioraron no solo al enlatado sino también a las uniones hechas de bejucos o material vegetal, resecándolas.

-Oxidación (27\%). Las uniones hechas con alambre o clavos se oxidaron con el tiempo por la exposición a la intemperie.

- Roturas (19\%) Las uniones hechas de bejucos se rompieron con las agresiones ambientales y humanas.

De acuerdo al porcentaje de recurrencia, las lesiones que predominan son las de tipo químico (Figura 13).

La causa que más originó deterioro en las uniones fueron las agresiones ambientales, y luego en menor grado las humedades. Otras causas que ocasionaron lesiones fueron debido a la característica propia del material y las agresiones de las personas. El proceso constructivo, la disposición de la unión en la construcción, los agentes bióticos, la falta de mantenimiento, las reparaciones inadecuadas, los problemas estructurales y las acciones accidentales y naturales no produjeron ninguna lesión en los diferentes tipos de uniones.

\subsection{Relleno}

El relleno, en general se encontró en buen estado. Presentaba lesiones cuando el empañetado está desprendido quedando al descubierto ante la intemperie.

\section{Las lesiones que prevalecieron fueron:}

- Roturas (96\%). Pérdida de partes de la pared debido a las agresiones ambientales, reparaciones inadecuadas o falta de mantenimiento. (Figura 14).

-Erosión atmosférica (54\%). Las acciones del viento y el sol erosionaron la superficie del relleno cuando estaba expuesto.

- Grietas (27\%). Aparecieron en el relleno por efecto de un esfuerzo excesivo o por reparaciones inadecuadas.

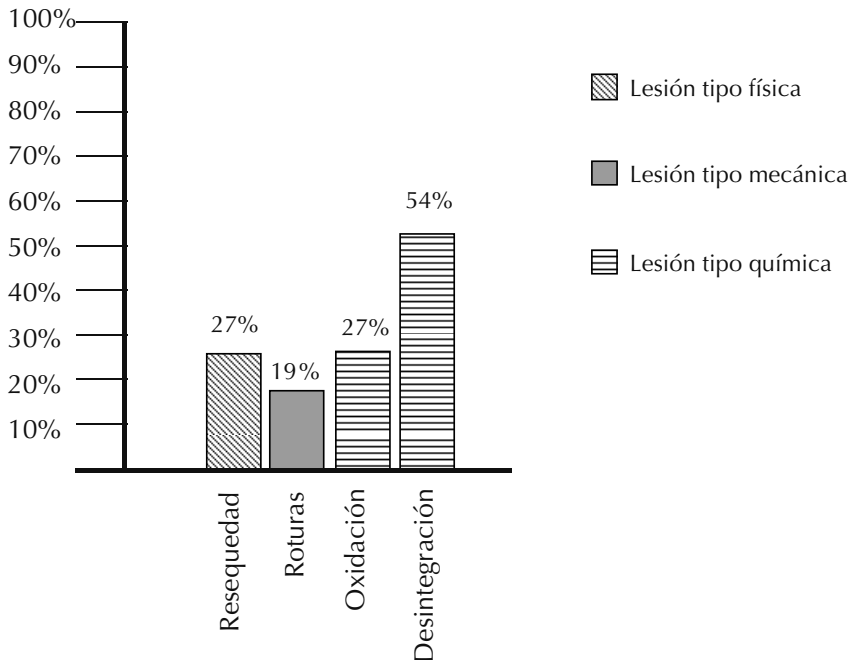

-Insectos xilófagos (15\%). Se observaron rastros dejados por insectos que se habían alojado en el relleno debido a la humedad. -Suciedad por depósito (8\%). El relleno mostraba suciedad proveniente de la lluvia y el viento.

- Humedad capilar (4\%). Por efecto de un alto nivel freático o por capilaridad estaba humedecida la parte inferior de la pared.

-Fisuras $(4 \%)$. Se observaron fisuras producto de un material mal amasado o por esfuerzos.

-Eflorescencia (4\%). Fue observada en muy pocos casos, producida probablemente por una humedad excesiva en el relleno.

-Mohos, musgos, líquenes (4\%). Fue detectada en la parte inferior del relleno posiblemente debido a mucha humedad en el relleno y en el ambiente.

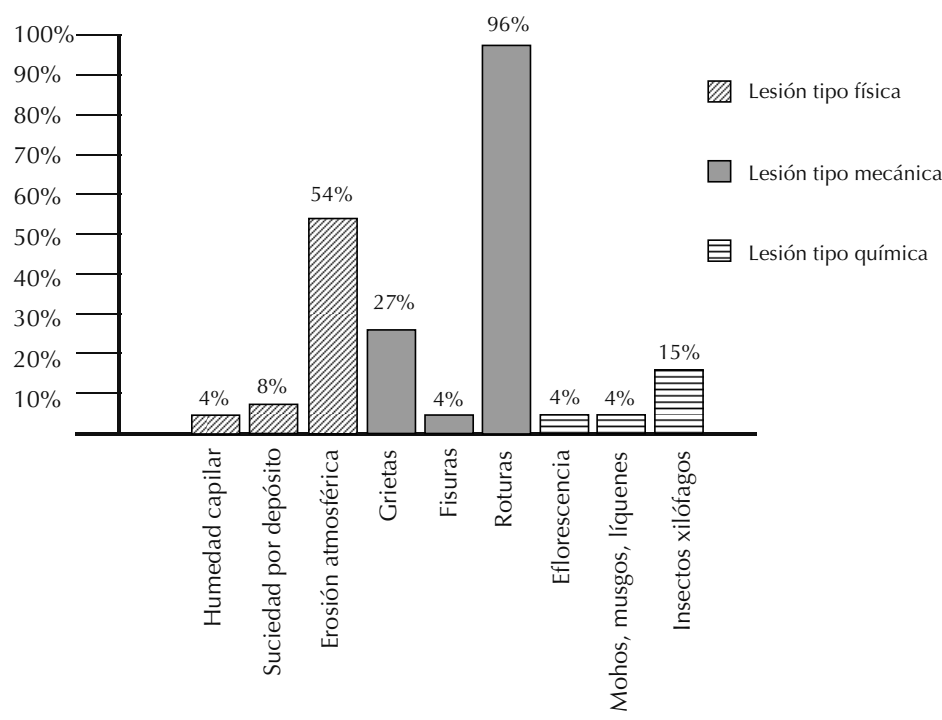

En el relleno, predominan porcentualmente las lesiones de tipo mecánico (Figura 15).

La causa que más afectó al relleno fueron las agresiones ambientales. Los problemas estructurales, las características del material de relleno, el proceso constructivo y la
13. Lesiones aparecidas en las uniones.

Elaboración de la autora.

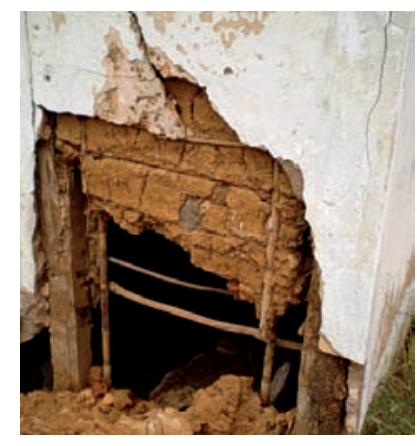

14 
16. Empañetado roto y descascarado - Vivienda en zona de Veritas en Maracaibo.

Archivo fotográfico de la autora (2009).

17. Lesiones aparecidas en el empañetado.

Elaboración de la autora.

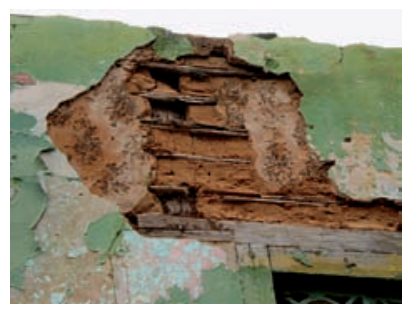

- Roturas (88\%). El empañetado estaba roto y no existía (Figura 16). Es la lesión predominante producto de los agentes ambientales y fue observada en todas las paredes exteriores.

-Desprendimientos del acabado (85\%). Es una de las lesiones más generalizadas y extendidas y fue observado en todas las paredes exteriores. Es producto de las agresiones ambientales, la falta de mantenimiento y reparaciones hechos con mortero de cemento.

-Suciedad por depósito (81\%). Las paredes exteriores se encontraban sucias, debido a los efectos del viento y de las lluvias y por falta de mantenimiento y limpieza.

-Descascaramiento (65\%). Fue observado más que todo en el acabado final (Figura 16). A lo largo del tiempo desaparecen las capas de pintura. Esta lesión es producto de la agresión ambiental, de la falta de mantenimiento y de las características propias del material usado en el acabado final.

-Grietas (58\%). producidas por reparaciones hechas con cemento u otro material poco compatible con el material del empañetado y en algunos casos por asentamientos de las paredes.

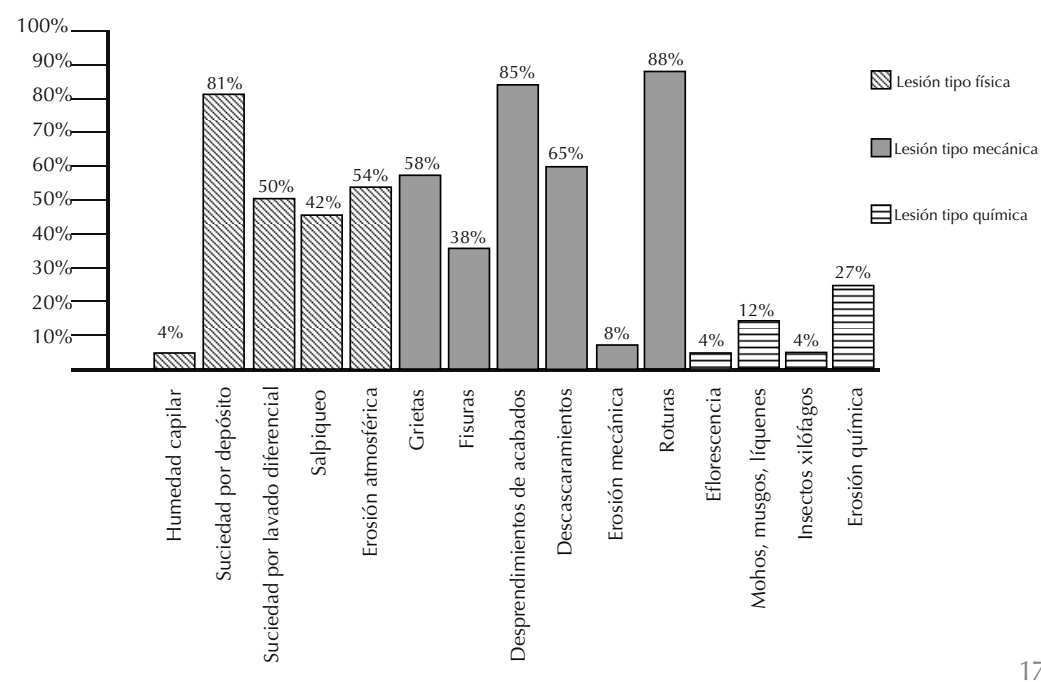

-Erosión atmosférica (54\%). El empañetado está todo erosionado por efectos de las Iluvias, del sol y del viento.

-Suciedad por lavado diferencial (50\%). Fueron observadas correntías de agua a lo largo de las paredes que dejaron marcas de suciedad. Son producto de las lluvias.

-Salpicaduras (42\%). Se encontraron en la parte inferior de las paredes exteriores, y se produce cuando la lluvia golpea la acera y las gotas salpican hacia los lados.

-Fisuras $(38 \%)$. Fueron producidas seguramente por dilataciones o contracciones del empañetado debido al material utilizado, por una mala aplicación y/o reparaciones con materiales incompatibles con el empañetado.

-Erosión química (ampollas) (27\%). Se observó el abombamiento de pintura, producto de humedad capilar o de la condensación o por utilizar pinturas a base de agua o aceite unas sobre otras o sobre el acabado de cal.

-Musgos, mohos, líquenes (12\%). Debido a la humedad excesiva se desarrollaron musgos y mohos en la parte inferior de algunas paredes internas.

- Humedad capilar (4\%). La parte inferior de la pared estaba mojada, producto de un alto nivel freático.

-Eflorescencia (4\%). Se observaron residuos de salitre en paredes internas que estaban húmedas.

- Insectos xilófagos (4\%). Esta lesión (rastros de insectos xilófagos) fue encontrada en una sola edificación.

En cuanto al porcentaje de recurrencia, las lesiones que más afectan el empañetado son de tipo mecánico y físico (Figura 17).

El empañetado fue la parte más afectada del bahareque. Las causas principales de su deterioro fueron las agresiones ambientales. Luego en menor medida la falta de mantenimiento, las características del material utilizado como empañetado, la disposición del empañetado en la pared, las humedades y las reparaciones inadecuadas. También hubo lesiones producto del proceso constructivo, las agresiones humanas, los agentes bióticos, por problemas estructurales y por acciones accidentales.

\subsection{De la pared completa}

Pandeo o desplome de pared, por efecto de asentamiento o por problemas de arriostramiento con el techo. Es una lesión de tipo mecánica.

\section{ALGUNAS RECOMENDACIONES PARA SU INTERVENCIÓN}

Para proponer algunas soluciones de intervención fueron revisadas una serie de expe- 
riencias practicadas en otros países, tales como: Viñuales (11), Doat y otros del grupo CRaterre (12), Maldonado y Vela (13), Minke (14), Hays y Matuk (15) y Pérez (16). También fueron estudiadas dos rehabilitaciones de paredes de bahareque que fueron ejecutados en el Estado Zulia en los últimos años, como fueron:

a) El proyecto de "Restauración de la capiIla de San Antonio", (6). Ejecutado por Arquiluz, C.A., Empresa rental de la Universidad del Zulia en el 2007, y

b) El estudio de "Recuperación de la Vivienda Natal de Rafael Urdaneta" (7) Ejecutado por Arquiluz, C.A. Empresa rental de la Universidad del Zulia en el 2007 (Figura 18)

Además fue construido por la autora (1), un prototipo de pared de bahareque aplicando varias de las soluciones propuestas en este artículo (Figura 19).

Por la naturaleza constructiva del bahareque no pueden intervenirse todos componentes al mismo tiempo, sino que cada uno debe ser intervenido siguiendo la secuencia de acuerdo al proceso constructivo de la técnica. Los componentes del bahareque: fundaciones, horconadura, enlatado, relleno y empañetado, son hechos de materiales diferentes, por lo que son requeridas distintas soluciones de intervención para cada uno de esos materiales. A continuación se proponen algunas recomendaciones para la intervención de una pared de bahareque, adicionándole algunos materiales o aditivos con la finalidad de aumentar su durabilidad y disminuir las lesiones que sufre.

\subsection{Fundaciones}

La degradación o pérdida de material en la parte inferior del horcón que va enterrado, puede ser reparado con acciones tendientes a prevenir la humedad, pudiendo ser algunas de ellas las siguientes:

Antes de iniciar con la reparación de las paredes, colocar elementos de soporte en ambas caras de la pared para evitar su colapso.

- Remover la parte podrida o degradada de los horcones y hacer una prótesis de madera (ver más adelante en horconadura). - Impermeabilizar la parte inferior del horcón con brea o cemento asfáltico hasta una altura aproximada de $80 \mathrm{~cm}$.

- Garantizar una buena fundación. Para esto puede hacerse una fundación corrida o fundaciones individuales o zapatas cuyas medidas dependerán del cálculo estructural y/o de las características de los suelos.
- Embutir el horcón hasta el nivel de piso dentro de una base de concreto. (Figura 20) -En lugares con alto nivel freático es recomendable construir una zanja filtrante perimetral cuya medida puede ser de $50 \mathrm{~cm}$ de ancho y $1 \mathrm{~m}$ de profundidad. Esta zanja permitirá evacuar las aguas de lluvias y evaporar el agua del nivel freático. Esta zanja debería realizarse de concreto mezclado con un aditivo hidrófugo. Tanto en las paredes como en el fondo de la zanja colocar tubos de 1" para el drenaje o una tubería con huecos que lleve el agua a un sumidero o espejo de agua cercano. Por último, rellenar toda la zanja con piedras de granzón de 1".

-Cuando las edificaciones presenten fundaciones aisladas, corridas o de mampostería de piedra, es recomendable que éstas sean frisadas con un producto impermeabilizante.

-En los casos que sea necesario, impermeabilizar la parte inferior de la pared con manto asfáltico. También pueden colocarse barreras impermeabilizantes debajo de las paredes usando para ello cualquier tipo de plástico.

\subsection{Horconadura}

- Retirar todos los horcones en mal estado. - Los horcones que solo presentan lesiones en la parte inferior, quitar la parte deteriorada y colocarles una prótesis. La prótesis debe ser con una madera igual o similar a la existente. La unión entre el horcón viejo y la prótesis puede ser a media madera, usando pernos para su fijación.

-Todos los horcones tanto viejos como nuevos deberían ser curados con un producto contra el ataque de insectos xilófagos, pudiendo usarse el aceite quemado, el gasoil o productos químicos especializados.

- Hacer muescas en el horcón o envolverlo con mecate o soga para mejorar la adherencia de la mezcla de barro a la horconadura.

\subsection{Enlatado}

Para el enlatado se recomienda lo siguiente:

- Dejar tal cual, el enlatado que está cubierto y retirar el enlatado que estaba al descubierto y se encuentra dañado.

- Hacer un nuevo enlatado con varas del material más asequible. Pueden ser de caña brava, bambú, ramas de árboles cercanos o listones de madera.

- Es recomendable tratar las varas con un baño de gasoil, aceite quemado y/o un producto químico anti-xilófagos.

- Las varas pueden ser amarradas una por una a cada lado del horcón, según la técnica tradicional (Figura 21) o puede hacerse una retícula prefabricada con las varas.
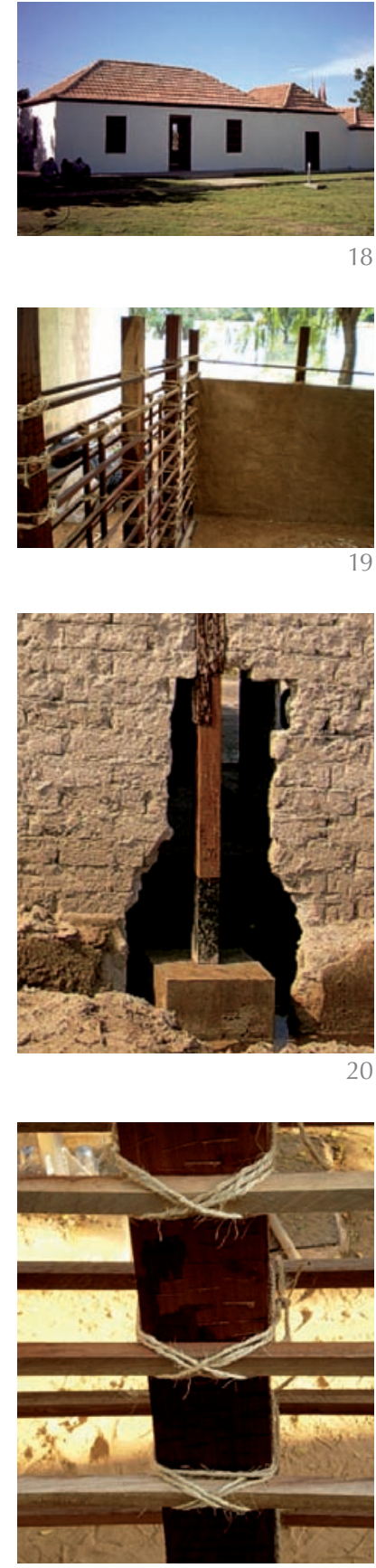

21

18. Vivienda Natal de Rafael Urdaneta después de la rehabilitación - El Carmelo, corresponde con la Figura 7.

Archivo fotográfico de la autora (2007).

19. Prototipo de pared de bahareque en construcción realizado por la autora.

Archivo fotográfico de la autora (2009).

20. Horcón con base de concreto - Rehabilitación Capilla en San Antonio.

Archivo fotográfico de la autora (2006).

21. Enlatado con amarre cruzado - Prototipo de pared de bahareque hecha por autora.

Archivo fotográfico de la autora (2009). 

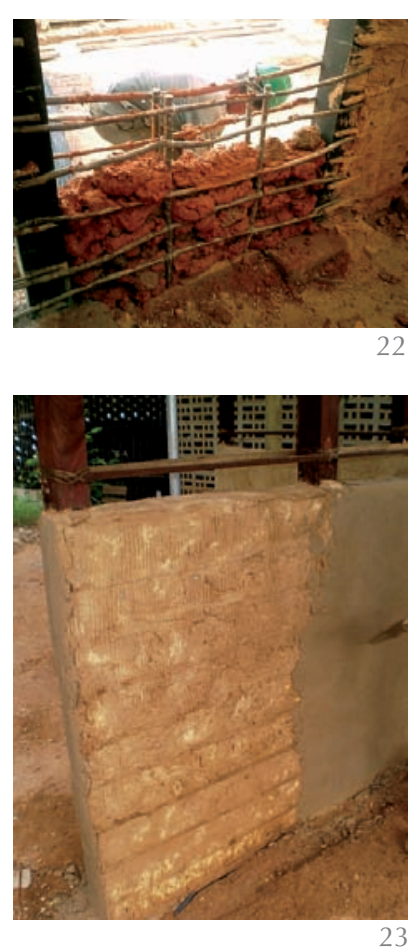

22. Rellenando con mezcla de barro y cal - Rehabilitación Vivienda Natal R. Urdaneta en El Carmelo.

Archivo fotográfico de la autora (2007).

23. Empañetado base y empañetado final o enlucido - Prototipo de pared de bahareque hecha por la autora.

Archivo fotográfico de la autora (2009).
- En aquellas partes donde la pared puede ser recuperada y donde quede al descubierto el enlatado original, se puede colocar encima las varas nuevas cortadas a la mitad en todo su largo y empalmarlas con el enlatado nuevo. De esta manera la pared vieja queda asegurada y hay continuidad en las varas.

- Para las uniones entre las varas y entre las varas y el horcón, lo mejor es utilizar materiales de fibras vegetales, mecate o mecatillo (Figura 21).

- Las distancias verticales entre las varas hacerlas entre 12 y $15 \mathrm{~cm}$.

- Amarrar al enlatado varas verticales auxiliares que estén ubicadas de forma equidistante entre los horcones para evitar el abombamiento.

\subsection{Relleno}

En cuanto a la mezcla del relleno, los materiales a usarse pueden ser variados, siempre y cuando contengan barro y piedras u otro material que le otorgue volumen a la mezcla como conchas de coco, escombros de bloques de ladrillos, restos de tejas, restos de baldosas, etc.

-En los primeros $60 \mathrm{~cm}$ a $80 \mathrm{~cm}$ desde el piso acabado, es recomendable colocar una mezcla con una gran proporción de piedras en ella, a manera de sobrecimiento. Pueden reutilizarse las piedras existentes de la pared original, después de haber sido lavadas y tratadas. También pueden usarse pedazos o escombros de bloques de cemento y/o arcilla, piedras, conchas de cocos, etc.

- La mezcla puede ser a base de arcilla (18\%), arena roja (72\%) y agua con bajas proporciones de cemento $y /$ o cal $(10 \%)$ para hacer una mezcla más resistente (Figura 22).

-Después de mezclar y amasar bien los materiales, éstos deben ser colocados en forma de bola dentro del enlatado, combinándolo con las piedras, conchas de coco u otro material duro.

- En lo posible conservar el relleno existente. Si esto fuera el caso, el relleno viejo debe estar bien húmedo antes de comenzar a colocar el relleno nuevo. Además se recomienda hacer unos orificios en el relleno viejo para que esos queden llenos con la mezcla nueva y mejorar la adherencia entre lo viejo y lo nuevo.

\subsection{Empañetado}

Para la reparación del empañetado deberían considerarse las siguientes acciones:

-Al iniciar la intervención de las paredes, el empañetado debe ser retirado con mucho cuidado y reparar una pared por vez. Como el empañetado es generalmente del mismo material que el relleno y queda completamente unido a él, al remover dicho empañetado en muchas ocasiones se producen desprendimientos también del relleno.

- Se debe realizar un empañetado base y un empañetado final o enlucido.

- Para el empañetado base puede usarse la misma mezcla que la del relleno y aplicarlo junto al relleno cubriendo el enlatado y la horconadura.

- Para que el empañetado final tenga buena adherencia con el empañetado base, este último debe estar relativamente rugoso. También pueden colocarse encima del empañetado base, piedrecitas para crear una textura rugosa.

- Dejar secar de 20 a 30 días antes de colocar el empañetado final.

- Colocar un empañetado final sobre el empañetado base que debe estar bien húmedo. Este empañetado final puede tener la misma mezcla que la del empañetado base o puede ser diferente, siempre y cuando sea compatible.

- Para el empañetado final puede usarse una mezcla hecha de arcilla $(9 \%)$, arena $(76 \%)$, cal en polvo $(12 \%)$, un poco de cemento (3\%) y agua (Figura 23 ).

- Esta mezcla debe ser aplicada en una sola capa de $2 \mathrm{cms}$, usando una Ilana hasta que quede lisa.

- Como protección se puede aplicar un acabado final consistente en una lechada de cal que debe ser renovada cada 6 meses o pintura látex.

- En caso de que el empañetado esté en buen estado, aplicar una ligera capa del empañetado final para que exista homogeneidad con el resto de las paredes. En estos casos repicar el empañetado y humedecerlo muy bien.

- Se puede hacer un rodapié de $20 \mathrm{~cm}$ usando una mezcla de cal $(44,5 \%)$, agua $(44,5 \%)$ y pegamento blanco $(11 \%)$.

\subsection{Reparación de lesiones}

Al realizar un proyecto de intervención igual como dentro de todo el proceso constructivo, debe garantizarse que las causas de las lesiones sufridas por la pared de bahareque sean eliminadas. Algunas recomendaciones al respecto son:

\subsubsection{Contra los agentes atmosféricos}

- Hacer las aislaciones hidrófugas en fundaciones y en bases de la pared, colocar grandes aleros y buenos drenajes en los techos y en el piso.

- Construir una acera alrededor de la edificación de por lo menos $30 \mathrm{~cm}$ de ancho y de 10 a $15 \mathrm{cms}$ de alto. Si es posible subir el nivel del piso de la edificación otro tanto. 
-En caso de que el nivel freático sea muy alto o que la edificación se encuentre en surcos de agua, se debe construir alrededor de la edificación una zanja de drenaje cuyas características variarán en los diferentes casos.

\subsubsection{Grietas y fisuras}

- Para arreglar grietas existentes, hacer llaves, preferiblemente de madera y luego volver a empañetar.

\subsection{Otras acciones}

-Antes de iniciar el proceso constructivo de cualquier intervención hay que cubrir toda la obra con lonas para protegerla de las posibles lluvias igual como apuntalar las paredes que van a ser intervenidas.

- Garantizar que la edificación tenga ventilación cruzada en todos los espacios y que las ventanas puedan abrirse de forma manual.

- Considerar protecciones solares para que la luz que penetre a los espacios sea de forma indirecta.

-Cualquier instalación de detección y prevención de incendios, de aire acondicionado, tuberías de aguas blancas y servidas, etc. deben respetar las características de la construcción de bahareque y estar ubicados en lugares en donde no afecten o dañen a la construcción.

- Hay que tener en cuenta todas las recomendaciones para la sismorresistencia a la hora de diseñar un proyecto de intervención.

- Cumplir con las generalidades de la normativa nacional de construcciones las cuales puedan aplicarse.

- Para conservar las edificaciones de bahareque hay que realizar un continuo mantenimiento al empañetado, que es la parte que más se deteriora. Para ello se aconseja realizar: limpieza periódica de las paredes, pintar cada 6 meses con el mismo material usado para el acabado final de la pared: pintura látex o lechada de cal, reparar con la misma mezcla cualquier desprendimiento, rotura, fisura o grieta que muestre el empañetado.

\section{CONCLUSIONES}

Esta investigación arrojó una serie de resultados y conclusiones que pueden ser enunciados como sigue:

1. Se pudo determinar, de forma aproximada, que el número de lesiones va decreciendo correlativamente en el orden inverso a la ejecución de la pared de bahareque, es decir, el componente más lesionado es el empañetado y aca- bado final con quince (15) tipos de lesiones, luego viene el relleno y el enlatado con nueve (9) tipos de lesiones, después la horconadura con ocho (8) tipos de lesiones y por último la parte del horcón que se encuentra enterrada con un (1) tipo de lesión. Hay que aclarar que las lesiones del relleno, enlatado y horconadura solo se producen cuando el empañetado esta desprendido o roto, quedando estas partes al descubierto y expuestas al ambiente. Cuando el empañetado está bien conservado, los otros componentes del bahareque generalmente se encuentran también en buen estado. Por lo tanto, es el empañetado y acabado final los que necesitan de mayor atención, cuidado y mantenimiento.

2. Aunque existen lesiones comunes a todos los componentes del bahareque, también hay lesiones completamente diferentes en cada uno de ellos; y estas diversas lesiones se presentan debido a los diferentes materiales que son usados en dichos componentes.

3. De las causas recurrentes que producen lesiones en el bahareque se encuentran las agresiones ambientales (sol, viento y Iluvias) igual como las humedades. Por lo que cualquier acción de intervención debe primordialmente contemplar la protección de las fachadas ante la intemperie con materiales resistente y la utilización de grandes aleros.

4. En cuanto al tipo de lesiones que sufre el bahareque estas son mayoritariamente de tipo físico y mecánico. Como este tipo de lesiones no cambian las propiedades de los materiales, como es el caso de las lesiones químicas, son de fácil reparación.

5. Actualmente es posible intervenir una pared de bahareque usando la misma técnica tradicional y utilizando materiales que pueden ser adquiridos en el mercado.

6. Aunque las intervenciones realizadas a las edificaciones suelen ser diferentes entre sí, ya que cada edificación como unidad constructiva requiere de una atención particular, muchas de las acciones de intervención y reparación a aplicar en las paredes de bahareque pueden generalizarse.

7. Cuando las paredes de bahareque se degradan, los usuarios generalmente desconocen la forma más óptima para su reparación, por lo que esta técnica ha comenzado a desvalorizarse en las áreas urbanas y sub-urbanas del estado Zulia, Venezuela. El estudio sobre las causas de las lesiones que sufren las paredes de bahareque y la manera como pueden ser reparados, no solo revalorizaría al bahareque sino que pudiese ser considerado como una opción técnico-constructiva para la edificación de viviendas. 


\section{BIBLIOGRAFÍA}

(1) Henneberg de León, A.: Paredes de bahareque en el Estado Zulia. Estudio integral para su rehabilitación sostenible. Tesis doctoral no publicada. Facultad de Arquitectura y Diseño de la Universidad del Zulia. Maracaibo, Venezuela, 2010.

(2) Real academia española: Diccionario de la lengua española. Tomos 1 y 2. $22^{a}$ edición. Madrid, España: Editorial Espasa Calpe S.A. 2001.

(3) Gasparini, G. y Margolies, L.: Arquitectura de tierra cruda en Venezuela. Caracas, Venezuela; Gráficas Armitano. 1998

(4) Borges, J.: Manuales de autoconstrucción. 1. Bahareque prefabricado (PS-4). Programa Vivienda Rural Viviendas Prototipo para la Zona Montano Frio. Mérida, Venezuela. Convenio de cooperación para la investigación de la vivienda y los asentamientos rurales ULA, MSAS, CONICIT.1992.

(5) Andrade, R.: Bahareque. Guía para la construcción de viviendas. Sistema constructivo E.P.R.T.B.R.Y2. Caracas, Venezuela: Fondo Editorial Vivienda Popular. 1996.

(6) Facultad de Arquitectura y Diseño: Proyecto de Restauración de la capilla de San Antonio. Documento no publicado. Coordinación de Extensión de la Facultad de Arquitectura y Diseño de la Universidad del Zulia y CORPOZULIA, Maracaibo, Venezuela, 2005.

(7) RAN consultores y Arq. Luis Otero: Proyecto de Recuperación de la Vivienda Natal de Rafael Urdaneta. Documento no publicado. CARBOZULIA, Maracaibo, Venezuela, 2006.

(8) Monjo Carrió, J.: Patología de cerramientos y acabados arquitectónicos. Editorial MuniIla-Lería. Madrid, España, 2000.

(9) Lasheras, Félix: Patología de la madera. En Departamento de Construcción. Universidad Politécnica de Madrid (eds.), Tratado de Rehabilitación Tomo 3: Patología y técnicas de intervención. Elementos estructurales. (pp. 255-276). Madrid, España: Editorial Munilla-Lería. 1998.

(10) Lasheras, F.: "Algunos conceptos básicos en patología de la edificación", en Revista electrónica ReCoPaR n 1. Marzo 2006. Madrid, España, p. 22, 2006. Recuperado en abril de 2008, de www.recopar.com/pdf/RECOPAR-N1.pdf

(11) Viñuales, G. M.: Restauración de arquitectura de tierra. Editorial del instituto argentino de investigaciones de historia de la Arquitectura del urbanismo. Universidad Nacional de Tucumán, 1981.

(12) Doat, P.; Hays, A.; Houben, H.; Matuk, S. y Vitoux, F. del grupo CRAterre: Construir con tierra. Tomos I y II. Fondo Rotatorio Editorial. Bogotá, Colombia, 1990.

(13) Maldonado, L. y Vela, F.: Curso de construcción con tierra I. Técnicas y sistemas tradicionales. Instituto Juan de Herrera. Escuela Técnica Superior de Arquitectura de Madrid. Madrid, España, 1999.

(14) Minke, G.: Manual de construcción en tierra. Segunda edición. Editorial Fin de Siglo. Uruguay, 2005.

(15) Hays, A. y Matuk, S.: Recomendaciones para la elaboración de normas técnicas de edificación con técnicas mixtas de construcción con tierra. En Técnicas Mixtas de Construcción con Tierra. (pp. 121-350). PROTERRA-PROYECTO XIV. 6. HABYTED Subprograma XIV-Tecnología para Viviendas de Interés Social. Programa Iberoamericano de Ciencia y Tecnología para el Desarrollo (CYTED). Archivo virtual en CD. 2003.

(16) Pérez, A.: Sistema bahareque. Chiapas, México. Ficha 3.3. En Un techo para vivir. Programa Iberoamericano de Ciencia y Tecnología para el desarrollo. CYTED. Subprograma XIV. Proyecto: XIV. 3 y XIV.5 con techo. PROGRAMA 10 x 10. Edicions de la Universitat Politècnica de Catalunya, SL. Barcelona, España. En formato CD. 2005. 